\title{
Particle Flux Associated With Stochastic Processes
}

by

C. E. Newman, P. A. Sturrock and E: Tademaru

SUIPR Report No. 386

August 1970

Prepared under

Air Force Office of Scientific Research

Contract F44620-69-C-0008

and

National Aeronautics and Space Administration

Grant NGL-05-020-272

INSTITUTE FOR PLASMA RESEARCH

STANFORD UNIVERSITY, STANFORD, CALIFORNIA 
PARTICLE FLUX ASSOCIATED

WITH STOCHASTIC PROCESSES

by

C. E. Newman, P. A. Sturrock ${ }^{*}$

and E. Tademaru ${ }^{\dagger}$

August 1970

SUIPR Report No. 386

Prepared under

Air Force Office of Scientific Research

Contract F44620-69-C-0008

and

National Aeronautics and Space Administration

Grant NGL-05-020-272

Institute for Plasma Research

Stanford Electronics Laboratories

Stanford University Stanford, California

* Also Department of Applied Physics, Stanford University

$\dagger$ Now at the National Radio Astronomy Observatory, Charlottesville, Virginia 


\section{Abstract}

The Lagrange expansion, which may be used to derive the FokkerPlanck equation, is here used to derive the corresponding expression for the flux of particles subject to a stochastic scattering process. The coefficients which occur in this expression are, in general, not the same as the coefficients which occur in the Fokker-Planck equation itself. In the special case that the particle distribution involves only one independent variable, the particle flux is determined by the familiar Fokker-Planck coefficients. Evaluation of particle flux is of special interest in the study of stochastic acceleration . 
The behavior of particles moving under the influence of random force fields is of interest in astrophysics. One example is the heating and diffusion of charged particles in the solar wind (Barnes, 1967; Jokipii 1966), and another is the acceleration of charged particles by the Fermi-type process of stochastic acceleration (Hall and Sturrock, 1967; Melrose, 1969; Tsytovich, 1966). Under conditions which are normally accepted, such a process may be represented by a Fokker-Planck equation, which gives the formula for the time derivative of the particle distribution function. However, in interpreting various solutions of this equation, it is helpful to know the flux of particles--in real space, momentum space, or in energy, as is appropriate to the problem. The formula for the particle flux is simple, but appears not to be widely known. This paper therefore gives a brief derivation of this formula.

In an earlier publication (Sturrock, 1960; hereafter called paper I), it was shown that the Fokker-Planck equation may conveniently be derived from a generalization of the Lagrange expansion. This publication also presented a formula for particle flux, so it offers a convenient starting point for this paper.

We consider the distribution of particles in a space enumerated by variables $x_{r}, r=1, \ldots, n$. These may be spatial coordinates, components of velocity or momentum, or any combination thereof. The distribution function $f(x, t)$ has the property that $f(x, t) d^{n} x$ is the number of particles in the volume $d^{n} x$ centered on the position $x$ at time $t$. If, with the requirement that $d x$ be small compared with the scale determined by the gradient of $f, f d^{n} x$ is not a large number, it is necessary to interpret this number as an "expectation" value.

If, when $t$ changes to $t+\Delta t$, the particle which was at position $\mathrm{x}$ moves to position $\mathrm{x}+\Delta \mathrm{x}$, the distribution function at time $t+\Delta t$ is related to that at time $t$ by the Lagrange expansion. An appropriate 
change of notation of equation (3.6) of paper I leads' to

$f(x, t+\Delta t)=f(x, t)-\frac{\partial}{\partial x_{r}}\left(f(x, t) \Delta x_{r}\right)+1 / 2 \frac{\partial^{2}}{\partial x_{r} \partial x_{s}}\left(f(x, t) \Delta x_{r} \Delta x_{s}\right)-\ldots$

The Fokker-Planck equation is derived from equation (1) by assuming that one may choose the time interval $\Delta t$ such that (a) the distribution function $f$ changes only slightly in this time; (b) the associated values of $\Delta x_{r}$ are small compared with the scale determined by the gradient of $f$; and (c) that a large number of random processes occur in this time with the result that

$$
\left.<\Delta \mathrm{x}_{\mathbf{r}}\right\rangle=O(\Delta t),\left\langle\Delta \mathrm{x}_{\mathbf{r}} \Delta \mathrm{x}_{\mathrm{s}}\right\rangle=O(\Delta \mathrm{t}),\left\langle\Delta \mathrm{x}_{\mathrm{r}} \Delta \mathrm{x}_{\mathrm{s}} \Delta \mathrm{x}_{\mathrm{t}}\right\rangle=\mathrm{o}(\Delta \mathrm{t}) \text {, etc. }
$$

where angular brackets denote the expectation value of a quantity. When the time interval $\Delta t$ is chosen in this way, equation (1) takes the usual form of the Fokker-Planck equation:

$$
\frac{\partial f}{\partial t}=-\frac{\partial}{\partial x_{r}}\left(f\left\langle\frac{\Delta x_{r}}{\Delta t}\right\rangle\right)+1 / 2 \frac{\partial^{2}}{\partial x_{r} \partial x_{s}}\left(f\left(\frac{\Delta x_{r}{ }_{r} x_{s}}{\Delta t}\right\rangle\right):
$$

Note that the term $\Delta \mathrm{x}_{r} / \Delta t$ may represent the effect of a small steady force field as well as that of a randomly fluctuating force field.

We now wish to obtain an expression for the flux $F_{r}$, the derivation of which is to be analogous to the derivation of equation (3). The same change of notation in equation (3.9) of paper I gives the following expression for the averaged flux:

$$
F_{r}=f\left\langle\frac{\Delta x_{r}}{\Delta t}\right\rangle-\frac{\partial}{\partial x_{s}}\left(f\left\langle\frac{d \Delta x_{r}}{d t} \Delta x_{S}\right\rangle\right)
$$


We see that, although the first term on the right-hand side of equation (4) also appears in the Fokker-Planck equation (equation 3), the second term does not. Nevertheless, we may confirm that the continuity equation

$$
\frac{\partial f}{\partial t}+\frac{\partial F}{\partial x_{r}}=0
$$

is equivalent to equation (3).

For this purpose, it is convenient to rewrite the flux as

$$
F_{r}=f\left(\frac{\Delta x_{r}^{r}}{\Delta t}\right)-\frac{\partial}{\partial x_{s}}\left(f \Gamma_{r s}\right)
$$

where

$$
\Gamma_{r s}=\left\langle\frac{d \Delta x_{r}}{d t} \Delta x_{s}\right\rangle \text {. }
$$

This may be expressed as

$$
\Gamma_{r s}=\Gamma_{\underline{r s}}+\Gamma_{r s}
$$

where $\Gamma_{\text {rs }}$ and $\Gamma_{\text {rs }}$ are symmetric and antisymmetric, respectively, in their suffixes. The symmetric component is expressible as a total derivative and may therefore be related to the second term of the FokkerPlanck equation:

$\Gamma_{\underline{r s}}=1 / 2\left\langle\frac{d \Delta x_{r}}{d t} \Delta x_{s}+\Delta x_{r} \frac{d \Delta x_{s}}{d t}\right\rangle=1 / 2\left\langle\frac{d}{d t}\left(\Delta x_{r} \Delta x_{s}\right)\right\rangle=1 / 2\left\langle\frac{\Delta x_{r} x_{s}}{\Delta t}\right\rangle$.

The antisymmetric term, given by

$$
\Gamma_{r s}=1 / 2\left\langle\frac{d \Delta x_{r}}{d t} \Delta x_{s}-\Delta x_{r} \frac{d \Delta x_{s}}{d t}\right\rangle
$$


does not appear in the Fokker-Planck equation because the term

$\frac{\partial}{\partial x_{S}}\left(f \Gamma_{r S}\right)$ is divergence free. Substitution of formula (4) into equation (5) therefore yields equation (3).

We see that, in general, the particle flux cannot be expressed in terms of the coefficients of the Fokker-Planck equation alone but in addition requires coefficients of the form $\left\langle\frac{d \Delta x_{r}}{d t} \Delta x_{s}\right\rangle$, which are readily calculated in the same manner as the usual Fokker-Planck coefficients. An important exception to this rule is the case that the system has only one independent coordinate. If, for instance, we are concerned only with the energy $\mathbf{E}$ of particles, the Fokker-Planck equation has the form

$$
\frac{\partial f}{\partial t}=-\frac{\partial}{\partial \mathbf{E}}\left(\mathrm{f}\left\langle\frac{\Delta \mathrm{E}}{\Delta t}\right\rangle\right)+1 / 2 \frac{\partial^{2}}{\partial \mathbf{E}^{2}}\left(\mathrm{f}\left\langle\frac{(\Delta \mathbf{E})^{2}}{\Delta t}\right\rangle\right)
$$

and the flux (in energy) is given by

$$
F_{E}=f\left(\frac{\Delta E}{\Delta t}\right)-1 / 2 \frac{\partial}{\partial E}\left(f\left(\frac{(\Delta E)^{2}}{\Delta t}\right)\right),
$$

with no additional terms.

This formula is of special interest for discussion of stochastic acceleration, since it is important to know whether a particular solution of the Fokker-Planck equation represents transfer of particles from low energy to high energy (acceleration) or from high energy to low energy (deceleration). This consideration has special relevance to a recent article by Melrose (1969) as will be discussed in more detail in a separate article (Tademaru, Newman and Jones, 1970). 


\section{ACKNOWLEDGEMENT}

The authors (E. T. and C. E. N.) acknowledge an interesting correspondence with Dr. D. B. Melrose. This work has been supported by the Air Force Office of Scientific Research under contract F 44620-69-C 0008 and the National Aeronautics and Space Administration under grant NGL-05-020-272. 


\section{References}

Barnes, A.: 1967, Phys. Fluids 10, 2427.

Hall, D. E. and Sturrock, P. A.: 1967, Phys. Fluids 10, 2620.

Jokipii, J. R.: 1966, Astrophys. J. 146, 480 .

Melrose, D. B.: 1969, Astrophys. Space Sci. 5, 181.

Sturrock, P. A.: 1960, J. Math. Phys. 1, 405.

Tademaru, E., Newman, C. E. and Jones, F. C.: 1970, Astrophys. Space Sci. (to be published).

Tsytovich, V. N.: 1966, Soviet Phys, Uspekhi 9, 370. 